\title{
EXERCICES DE PHILOSOPHIE LITTÉRAIRE *
}

Articles réécrits ou études inédites, ces « exercices de philosophie littéraire » aboutissent à un grand livre, très maitrisé, plein de savoir et de talent. D'une écriture précise et patiente, subtile et lumineuse, évasive parfois, mais le plus souvent profonde sans obscurité et démonstrative sans simplisme, $A$ quoi pense la littérature s'adresse, sans parade ni affeterie, à tous ceux qu'intéressent les rapports du philosophique et du littéraire, autrement dit le travail " des idées dans les lettres" (p. 200). L'auteur y poursuit une réflexion, engagée il y a près de trente ans, sur les devenirs croisès de l'entreprise philosophique et de l'activité littéraire dans la France des $\mathrm{XIX}^{e}$ et $x x^{e}$ siècles. Pierre Macherey, on le sait, a été partie prenante du mouvement de relecture de Marx dont Louis Althusser fut l'initiateur aussi vite contesté que reconnu. Co-auteur de Lire Le Capital ${ }^{1}$, P. Macherey publiait, dès 1966, un ouvrage ambitieux intitulé Pour une théorie de la production littéraire'.

Souvenons-nous. C'était, deux ans avant mai 68 , la pleine vogue du structuralisme. Au zénith brillait la trinité Barthes-Lacan-Lévi-Strauss, à laquelle on amalgamait Althusser doublé de Foucault (l'anti-humanisme) et bientôt Derrida flanqué de Kristeva (l'anti-historicisme). Déjà retombaient, non sans conséquences sur l'enseignement des lettres, les éclats de la Querelle des deux Critiques (Picard contre Barthes). À ces belligérants, tous deux tributaires de la même question essentialiste : "Qu'est-ce que la littérature?", Pour une théorie de la production littéraire opposait l'exigence d'un questionnement matérialiste : « $\AA$ quelles conditions réelles faut-il rapporter la production et le fonctionnement du discours littéraire? " Affaibli par son théoricisme et par le flou de certaines formulations, mais racheté par son goût bien concret des textes, ce livre inégal et vigoureux exposait " quelques concepts élémentaires ", confrontait des pratiques critiques (Lénine lecteur de Tolstoĩ face à l'analyse structurale) et proposait des lectures originales de J. Verne et de Balzac. Penser le travail du littéraire sur l'idéologique; chercher dans la littérature un analyseur de la pensée sociale d'une époque;

* À propos de Pierre Macherey, A quoi pense la littérature? Exercices de phllosophie littéraire. Paris, Presses universitaires de France, 1990. 14,5 $\times 21,5,253$ p. (« Pratiques théoriques ").

1. Paris, Maspero, 1965.

2. Paris, Maspero, 1966.

Revue de synthèse : IV S. Nos 1-2, janv.-juin 1992. 
éprouver la pertinence du concept de « décalage » appliqué aux conflits dont se nourrit une cuvre littéraire toujours «décentrée, disparate, déterminée, complexe "... Ces ambitions, P. Macherey était, en 1966, le premier à les formuler aussi nettement et à tenter d'y faire face. Éclipsé, après 1968, par des avant-gardes plus voyantes - de Tel Quel à H. Meschonnic - cet effort a trouvé un prolongement avec les travaux - bien distincts par l'objet et la méthode - de Renée Balibar dont P. Macherey préfaçait Les Français fictifs : le rapport des styles littéraires au français national ${ }^{3}$ que sont venus étayer L'Institution du français : essai sur le colinguisme des Carolingiens à la République ${ }^{4}$ et, toute récente, une peu banale Histoire de la littérature française 5 . L'un et l'autre entendent repenser l'activité littéraire à partir de ses conditions historiques de possibilité : chez $\mathbf{R}$. Balibar, la notion de colinguisme conceptualise tout un jeu d'interactions entre langues et littératures européennes; chez P. Macherey, la littérature s'inscrit dans de grandes configurations culturelles désignées en termes althussériens ou, plus récemment, par référence à la notion d' « épistémé » empruntée à Foucault.

A quoi pense la littérature? recoupe, pour sa part, deux séries de questions qui appartiennent en propre à la recherche philosophique. D'abord, une confrontation du spinozisme et de l'hégélianisme ${ }^{6}$. Ensuite, une exploration de l'histoire de la "philosophie à la française », des Idéologues à Bergson, pour y mesurer les incidences culturelles de la bifurcation neuve qui s'opère, au xix siècle, entre science et littérature. Car cette séparation renvoie elle-même à un partage plus ancien. Dans l'Europe de la fin du xvir' siècle - de Diderot à Kant et sous des formes nationales diverses - littérature et philosophie se délimitent et s'autonomisent l'une par rapport à l'autre. En France, l'appareil scolaire érige chacune en discipline distincte. Il en résulte, pour l'enseignement de la philosophie, un équilibre instable entre son ancrage scientifique et son marquage littéraire, ainsi que - en cet âge de la Littérature, qui se réclame aussi de la Science - l'émergence d'un entre-deux propice au dialogue entre écrivains et philosophes et au brassage intertextuel des idées. Au même moment, Balzac et Comte cherchent "l'unité de composition" du tout social; Zola, après Schopenhauer, voit le monde sous l'aspect d'une volonté aveugle; plus tard, Queneau, complice de Kojève, exploite ironiquement le thème de la fin de l'histoire... De ces connivences, Macherey analyse finement les manifestations textuelles.

Ce philosophe à l'écoute de la littérature marque ses distances par rapport à deux positions inverses qu'il récuse d'un même geste, analogue à la démarche de G. Deleuze dans son superbe Proust et les signes ${ }^{7}$. Le primat traditionnel du « logos » sur le « muthos » postule la suprématie d'une philosophie qui s'attribue le privilège d'être le non-encore-pensé d'une littérature réduite, dès lors, au rôle illustratif d'une littérature d'idées, voire d'une littérature à thèse. Sous la bannière avant-gardiste de la déconstruction et du pan-textualisme, la position opposée

3. Paris, Hachette, 1974.

4. Paris, Presses universitaires de France, 1985.

5. Paris, Presses universitaires de France, « Que sais-je ? », 1991.

6. Cf. Pierre Macherey, Hegel ou Spinoza, Paris, Maspero, 1979.

7. Paris, Presses universitaires de France, 1976. 
consiste à faire de la littérature le refoulé, c'est-à-dire la vérité d'une philosophie qui, coupée de sa relation avec le discours scientifique, n'est plus alors qu'une "scène fabuleuse " (Derrida). Aux yeux de P. Macherey, nulle précellence, mais un mélange sans cesse à démêler et des limites qui ne sont pas des frontières, mais une commune et réciproque limitation. La notion de "philosophie littéraire" revient donc à considérer qu'avec les moyens qui lui sont propres, la littérature produit de la pensée, qu'elle a valeur d'expérience de pensée. D'où le choix du titre. Non pas : "Que pense la littérature? ", ce qui réduirait cette pensée à un contenu systématisable et séparable des formes rhétoriques qui lui donnent force et figure. Mais : « À quoi pense la littérature? », c'est-à-dire à quoi peut-elle faire penser les lecteurs assez vigilants pour capter cette pensée sans concepts, cette pensée par figures et métaphores que la littérature, en ses formes mêmes, invente et diffuse? " Machine à penser » ou " opérateur spéculatif », entretenant avec la philosophie un rapport critique, ironique, ludique, la littérature suscite, en même temps que le plaisir de sa lecture, des effets de vérité, un savoir spécifique que Macherey qualifie de " philosophie spontanée des écrivains » (p. 198) et, s'agissant d'une pensée qui excède les pouvoirs du sujet individuel, de « philosophie sans philosophes " (p. 196). Vérité et savoir subversifs par vocation, puisque le propre de la littérature, c'est de dire tout ce qu'il ne faudrait jamais dire (p. 203).

De son corpus principal, P. Macherey a préféré écarter des écrivains trop ouvertement philosophes, tels Proust ou Mallarmé, qu'il ne se prive d'ailleurs pas d'évoquer quand besoin est (voir p. 189 ou p. 244). À deux exceptions près, les textes qu'il a élus ne relèvent pas de la classe des chefs-d'œuvre consacrés. Par leurs dates, tous appartiennent à l'âge de la Littérature : le plus ancien a été écrit en 1784 (Les Cent vingt journées) et le plus récent, l'essai de Foucault sur Roussel, a été publié en $1963^{8}$. Ces neuf leçons de philosophie littéraire se développent à partir de neuf noms d'écrivains, eux-mêmes répartis entre trois séries thématiques dont les variations offrent un parcours spéculatif original, loin des chronologies et des typologies de l'histoire littéraire. Car il ne s'agit pas non plus de monographies sur des auteurs : au lieu d'isoler «la pensée de Sand» ou «la philosophie de Céline ", Macherey s'efforce de cerner, dans sa multiplicité intertextuelle, une pensée littéraire qui, traversant les noms propres, circule dans tout l'imaginaire d'une époque (p. 198-200). Staël, Sand, Queneau : la première série nous entraîne par « les chemins de l'histoire ", pour une méditation anthropologique sur les voies du devenir humain. Avec Hugo, Bataille et Céline, nous basculons " au fond des choses", dans une ontologie négative qu'organisent les schèmes de l'immanence, du renversement, des profondeurs. La troisième visée est aussi l'ultime puisqu'elle débouche sur le vide et la mort; avec Sade, Flaubert et Roussel, une rhétorique lucidement folle déploie ses excès jusqu'aux limites du langage et de la pensée; désormais « tout doit disparaître »...

Sur ces neuf essais, étayés de notes inspirantes (p. 204-253), quelques mots de présentation... L'enthousiasme de Mme de Staël ouvre ces "chemins de l'histoire " que vient clore la dérision de R. Queneau. Héroïnes hybrides, rhétorique

8. Michel Foucault, Raymond Roussel, Paris, Gallimard, 1963. 
du composite, lecture ouverte de Kant et foi en une Allemagne réinventée, l'imaginaire cosmopolite de la baronne porte l'espérance d'une histoire réconciliant le national et l'universel. Chez G. Sand, les deux versions de Spiridion, roman panthéiste, attestent les influences croisées de Lammenais et Leroux; mais cette quête d'une religion de l'humanité passe par le travail de la fiction; et le panthéisme se fait machine à raconter. Avec Queneau, scribe des fameuses leçons de Kojève, c'est un conteur philosophe qui prémédite ses divagations hégéliennes, de Pierrot mon ami au Dimanche de la vie, pour aboutir - de mèche avec Volterra, inventeur italien de la biométrie - au constat que « l'histoire est la science du malheur des hommes »... Par son désir de révolution, c'est toute une époque qui veut aller " au fond des choses " (Marx) jusqu'à légitimer " l'homme d'en bas » cher à Sue, à Hugo, à Baudelaire, au fantastique social véhiculé par le feuilleton ; car si ces histoires souterraines renvoient à un imaginaire de la profondeur et du renversement partagé par Hegel ou Heine, Marx ou Tocqueville, c'est bien parce que la littérature se doit désormais d'inventer des systèmes de symbolisation aptes à répondre aux angoisses de ce siècle où Dieu est mort. Ce vertige du gouffre se retrouve chez Bataille qui, paradoxalement, préconise un dualisme axiologique pour revitaliser le matérialisme " par le bas ", au prix d'une rupture avec le surréalisme, mais d'abord avec l'économisme, car l'homme n'est pas un être de besoin, mais un animal symbolique : "l'homme est ce qui lui manque." Pour construire une rhétorique des abîmes, le styliste Céline invente son « petit truc " : la phrase parlée d'un locuteur obscène, sorte de métro magique qui transporte le lecteur, comme si quelqu'un lui « lisait dans la tête », lui disait tout ce qui ne doit pas être dit... Les trois embardées vers les limites démarrent par un superbe essai sur Sade que scandent ironiquement les trois questions kantiennes : que m'est-il permis d'espérer? le pouvoir que donne le crime; que dois-je faire? jouer le plaisir (le réel) contre le désir (la transcendance), donc tuer Dieu par une logique de l'abjection; que puis-je connaître? tous les possibles de cette abjection grâce à une machine narrative qui me mène à la vérité de l'homme : la mort. Lisant La Tentation de saint Antoine comme le Bildungsroman panthéiste d'un naïf assiégé par les images, P. Macherey y débusque non seulement le symbolisme primitif de Creuzer, mais des traces de Schopenhauer, de Spinoza relu à travers Hegel, des matérialistes allemands (Moleschott, Büchner, Häckel); pour connaître le bonheur d' " être la matière ", l'écrivain doit " aller au crottin " et tirer l'or littéraire du fumier d'un réel que le style restitue comme absence; car le style, qui fait " sonner le creux des mots ", néantise le réel jusqu'à n'en faire qu'une obsession, une " tentation ". À l'irréalisme de Flaubert répond celui d'un R. Roussel en qui le psychiatre Janet voulait ne voir qu'un malade mental; Foucault renverse la perspective : il y a une folie propre à l'œuvre, la folie de dire la totalité des choses ; du travail des mots se dégage une leçon d'ontologie sur le rapport des mots au réel; donc mieux vaut lire Roussel à la lumière de Saussure et de Mallarmé qu'à celle de Freud; la littérature, ascèse du dire, nous enseigne à voir les choses du point de vue de leur disparition; elle nous enseigne à mourir (p. 191).

Au terme de ces essais pénétrants - dont certains, sur Sade, Flaubert, Hugo, devraient faire date - on est tenté de poser à ce lecteur intrépide deux questions de méthode. La première porte sur le statut des rapports intertextuels. P. Macherey « rapproche » ses textes avec flair, dextérité et pertinence, en vertu d'une affi- 
nité thématique ou d'une cohérence synchronique. Mais il ne se soucie pas de codifier ni de définir sa démarche en comparaison avec d'autres possibles, que ce soit le dialogisme selon Bakhtine ou les approches formalistes (Genette) et herméneutiques (Starobinski). Ce qui conduit à une autre question. Fidèle au procès de l'idéalisme subjectif instruit par Althusser et Foucault ainsi qu'à sa propre déconstruction de l'illusion interprétative, P. Macherey récuse l'idée d'un secret de l'œuvre, d'un sens caché que viendrait révéler une conscience herméneutique souveraine (voir p. 8 et p. 194). Si motivé soit-il, ce procès autorise-t-il à frapper d'invalidité tout recours au concept d'interprétation et à évacuer, du même geste, le problème du sujet scripteur ou lecteur? Le propre de la littérature n'est-il pas de produire un discours qui permet à un sujet individuel d'interpréter, par un langage à fonction symbolique, tous les types de modélisation du savoir/du réel ${ }^{9}$ ?

Sans préjuger des réponses que Pierre Macherey pourrait, sur ces points, apporter, on se permettra in fine de saluer, pour son énergie, son alacrité, sa justesse chaleureuse, la subjectivité interprétative de l'auteur $\mathrm{d}^{\prime} \dot{A}$ quoi pense la littérature? Car l'usage de la littérature qu'il propose implicitement dans sa conclusion ( « De Sade à Céline, la littérature semble s'être vouée à l'exposition de tout ce qu'il ne faudrait pas dire [...] le monde renait plus vrai que nature, dans la lumière impitoyablement cruelle et cynique que projette sur lui la vérité d'un style ») nous invite à faire des livres - et d'abord de ce livre - un viatique pour temps difficiles, l'arme d'un gai savoir pour entrer dans le xxic siècle.

Éric WALter.

9. Cf. Claude Reichler, « La littérature comme interprétation ", in C. Reichler, dir., L'Interprétation des textes, Paris, Minuit, 1989. 